\title{
Erratum to: Determination of the Active Components in Chinese Herb Cortex Moutan by MEKC and LC
}

\author{
K. Yu $\cdot$ Y. W. Wang
}

Published online: 10 July 2011

(C) Springer-Verlag 2011

\section{Erratum to: Chromatographia (2006) 63:359-364 DOI: $10.1365 / \mathbf{s 1 0 3 3 7 - 0 0 6 - 0 7 6 0 - 7}$}

Regrettably, there are some mistakes in the original version of this article.

The authors were stated incorrectly. The correct authors are $\mathrm{K}$. Yu and Y. W. Wang, the corresponding author is $\mathrm{K}$. $\mathrm{Yu}$ and his affiliation is "Shanghai Institute of Materia Medica, Chinese Academy of Sciences, Shanghai 201203, China; email: yuk@mail.shcnc.ac.cn”.

On page 359 , paragraph 3 , line 26 , the sentence should be modified as "In our preview study, a MEKC method was preliminary developed for the analysis of $\mathrm{PN}$ and $\mathrm{PF}$ in Cortex Moutan and the related herbal product [1]. The aim of this study is to compare the analytical performance of
MEKC to HPLC for the quantification of the PN and PF." In the legends of Tables 2 and 3, " $(n=5)$ " is missing for MEKC. In "Method Validation of MEKC" section on page 362 , the text should correspondingly read "5 times", "5 samples", and "5 days". On page 363 , paragraph 4 , line 7 , the average recovery of MEKC method should be 97.6-104.0\% for PN and 95.3-103.8\% for PF. In Table 7, the value of PF determined by MEKC should be $11.54 \pm 0.68$.

\section{Reference}

1. Yu K, Wang YW, Cheng YY (2006) J Pharm Biomed Anal 40:1257-1262

The online version of the original article can be found under doi:10.1365/s10337-006-0760-7.

\section{K. Yu (ه)}

Shanghai Institute of Materia Medica,

Chinese Academy of Sciences, Shanghai 201203, China

e-mail: yuk@mail.shcnc.ac.cn

Y. W. Wang

Pharmaceutical Preparation Section, The Seventh People's

Hospital of Hangzhou, Hangzhou 310013, China 